\title{
Knots, Feynman Diagrams and Matrix Models
}

\author{
Martin Grothaus \\ BiBoS, Universität Bielefeld, D 33615 Bielefeld, Germany \\ Ludwig Streit \\ BiBoS, Universität Bielefeld, D 33615 Bielefeld, Germany \\ CCM, Universidade da Madeira, P 9000 Funchal, Portugal \\ Igor V. Volovich \\ Steklov Math. Inst., RAS, R 117966 Moscow, Russia
}

July 29, 2018

\begin{abstract}
An $U(N)$-invariant matrix model with $d$ matrix variables is studied. It was shown that in the limit $N \rightarrow \infty$ and $d \rightarrow 0$ the model describes the knot diagrams. We realize the free partition function of the matrix model as the generalized expectation of a Hida distribution $\Phi_{N, d}$. This enables us to give a mathematically rigorous meaning to the partition function with interaction. For the generalized function $\Phi_{N, d}$ we prove a Wick theorem and we derive explicit formulas for the propagators.
\end{abstract}




\section{Contents}

\begin{tabular}{lll}
\hline 1 & Introduction & 3
\end{tabular}

2 Gaussian analysis on matrix spaces $\quad 5$

2.1 Gaussian spaces on matrix spaces . . . . . . . . . . . . . . . 5

2.2 Generalized functions on matrix spaces . . . . . . . . . . . . . 8

3 Symplectic Feynman distributions $\quad 10$

3.1 One dimensional case . . . . . . . . . . . . . . . . . . . . . . . 11

3.2 Finite dimensional case . . . . . . . . . . . . . . . . . . . . . . 13

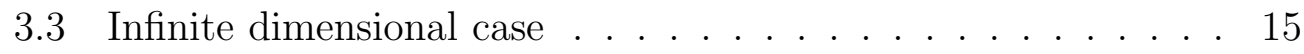

$\begin{array}{|lr|}4 \text { Proof of Theorem } & 17\end{array}$

4.1 Knots . . . . . . . . . . . . . . . . . . . . . . . . 17

4.2 Wick theorem and propagators . . . . . . . . . . . . . . . 19

4.3 Feynman diagrams . . . . . . . . . . . . . . . . . . . . . . 22

$\begin{array}{lll}5 & \text { Knots and M(atrix)-theory } & 26\end{array}$

\begin{tabular}{lr}
\hline References & 28
\end{tabular} 


\section{Introduction}

In this note we study an $U(N)$-invariant matrix model with the following partition function

$$
Z(N, d, g)=\int_{\mathbb{R}^{2 d N^{2}}} \exp (i S(A, B, g)) d A d B
$$

where the action is

$$
S(A, B, g)=\operatorname{Tr}\left(A_{\mu} B_{\mu}\right)+\frac{g}{2 N} \operatorname{Tr}\left(A_{\mu} B_{\nu} A_{\mu} B_{\nu}\right) .
$$

Here $N, d \in \mathbb{N}, A_{\mu}$ and $B_{\mu}$ are Hermitian $(N \times N)$-matrices over the field $\mathbb{C}$, $1 \leq \mu \leq d$. We assume the summation over the repeating indices and $g \in \mathbb{R}$ is the coupling constant. The measure

$$
d A:=\prod_{\mu=1}^{d}\left(\left(\prod_{1 \leq k \leq l \leq N} d \Re A_{\mu}^{k l}\right)\left(\prod_{1 \leq k<l \leq N} d \Im A_{\mu}^{k l}\right)\right) .
$$

Our investigations are motivated by a paper of Aref'eva and Volovich AV98 in which the authors proved the following theorem.

Theorem 1.1 The set of connected Feynman diagrams for the model (1) in the limit $N \rightarrow \infty$ and $d \rightarrow 0$ is in one-to-one correspondence with the set of alternating knot diagrams. The generating function for the alternating knot diagrams is given by the expression

$$
F(g)=\lim _{d \rightarrow 0} \lim _{N \rightarrow \infty} \frac{1}{d N^{2}} \ln Z(N, d, g) .
$$

Theorem 1.1 can be regarded is an application of quantum field theory to the theory of knots. Another approach has been performed by Witten Wit89. There the author has considered the Wilson loops in Chern-Simons gauge theory as knots. In general the path integrals used in quantum field theories are not mathematically well-defined. In [AS94 and Sch91] a rigorous mathematical meaning has been given to the path integral of the Abelian Chern-Simons model, using the theory of Fresnel integrals developed in AH76; and in [LS96 this has been done in the frame of white noise analysis, see [HKPS93]. Furthermore, in [LS96" the authors have specified the Wilson loops and have proved the relation to topological invariants which 
has been conjectured in Wit89. In the non-Abelian case a mathematically rigorous meaning to the Chern-Simons integral has been given in AS97, also by using the white noise approach.

The theory of knots, see Ati90 and Kau90, is used in low dimensional topology and also in physics, chemistry and, biology. Recently Faddeev and Niemi [FN97] have suggested that in certain relativistic quantum field theories knot-like configurations may appear as stable solitons. The remarkable progress in the classification of knots, based on Jones' and Vassiliev's invariants, has been related with the application of von Neumann algebras, Yang-Baxter equations, and singularity theory, for a review see [Bir93] and BNGRT97.

The large $N$ limit is considered in quantum chromodynamics (QCD), matrix models and superstring theory, see t'H74, BIPZ78, AAV96, |AV96, [BFSS97], [IKKT97], and [DVV97], the limit $d \rightarrow 0$ is considered in the theory of spin glasses and in polymer physics, see dG82 and Are83.

The statement of Theorem 1.1 reminds the matrix approach to superstring theory, see [BFSS97], [Per97], [IKKT97], and [DVV97], where spacetime is represented as the moduli space of vacuum and strings appear in the large $N$ limit. See also the concluding remarks given in Section 5 .

In order to prove Theorem 1.1, in AV98 the authors have expanded the partition function $Z$, see (1), into the formal perturbation series in the coupling constant $g$

$$
Z=\sum_{k=0}^{\infty} \frac{1}{k !}\left(\frac{i g}{n}\right)^{k} \int_{\mathbb{R}^{2 d n^{2}}}\left(\operatorname{Tr}\left(A_{\mu} B_{\nu} A_{\mu} B_{\nu}\right)\right)^{k} \exp \left(i \operatorname{Tr}\left(A_{\mu} B_{\mu}\right)\right) d A d B .
$$

Our contribution to the proof of Theorem 1.1 is to give a mathematically rigorous meaning to the Gaussian integrals in (2), to prove a Wick theorem for these integrals, and to derive explicit formulas for the propagators.

This paper is organized as follows. In Section 2 we introduce the concepts of Gaussian analysis as far as necessary for the constructions in this note. In doing so we concentrate on the special case in which the underlying nuclear triple is given by matrix spaces. In order to give a mathematical rigorous meaning to the integrals in (2) the spaces of generalized functions (Hida distributions) we introduce in Section 2.2 are essential. For a detailed exposition of Gaussian and white noise analysis we refer to the monographs [Hid80, BK95, HKPS93, Oba94, and Kuo96. In Section 3 we realize the partition function (1) for $g=0$ as the generalized expectation of a Hida 
distribution $\Phi_{N, d}$. At first we consider the one dimensional case, i.e., $N=1$, and then the case of an arbitrary $N \in \mathbb{N}$. In our first approach we obtain for different $N$ different spaces of Hida distributions. In order to calculate the large $N$ limit it turns out to useful to embed all $\Phi_{N, d}$ in a joint space of Hida distributions. This embedding we derive in Section 3.3. In Section 4 we discuss the proof of Theorem 1.1. First we shortly recall the necessary definitions and notions from the theory of knots. In Section 4.2 we give a mathematically rigorous definition of the Gaussian integrals in (2), see Definition 4.1, prove a Wick theorem for the generalized function $\Phi_{N, d}$, see Theorem 4.3, and derive explicit formulas for the propagators, see Theorem 4.5. Then we show how to apply the Feynman diagram technique in order to find an explicit formula for the logarithm of the partition function, see (24). This formula enables us to determine the limit $N \rightarrow \infty$ and $d \rightarrow 0$ of the normalized logarithm of the partition function and to prove Theorem 1.1. Finally, in Section 5 we quote some concluding remarks on the consequences of Theorem 1.1 for M(atrix)-theory given in AV98.

\section{Gaussian analysis on matrix spaces}

\subsection{Gaussian spaces on matrix spaces}

We start by considering a Gel'fand triple

$$
\mathcal{M} \subset \mathcal{T} \subset \mathcal{M}^{\prime}
$$

Here $\mathcal{T}$ is a real separable Hilbert space of matrices. Its inner product $(\cdot, \cdot)$ we assume to be given by the trace of the matrix product of two matrices and the corresponding norm we denote by $|\cdot|$. The matrix space $\mathcal{M}$ we assume to be a nuclear space densely topologically embedded in $\mathcal{T}$. Furthermore, we assume that $\mathcal{M}$ is the projective limit of a family of Hilbert spaces $\left(\mathcal{T}_{p}\right)_{p \in \mathcal{I}}$ parameterized by elements of an arbitrary set of indices $\mathcal{I}$ such that $\mathcal{M}=$ $\cap_{p \in \mathcal{I}} \mathcal{T}_{p}$ is dense in all $\mathcal{T}_{p}$ and that for all $p_{1}, p_{2} \in \mathcal{I}$ there exists $p \in \mathcal{I}$ such that $\mathcal{T}_{p} \subset \mathcal{T}_{p_{1}}$ and $\mathcal{T}_{p} \subset \mathcal{T}_{p_{2}}$ and the embeddings are of Hilbert-Schmidt class. Let $\mathcal{T}_{-p}$ be the dual of $\mathcal{T}_{p}$ w.r.t. the Hilbert space $\mathcal{T}$ (if it makes no sense to consider $-p$ of $p \in \mathcal{I}$ this notation is symbolically and means that $\mathcal{T}_{-p}$ is the negative space corresponding to $\mathcal{T}_{p}$, see [BK95]). Then $\mathcal{M}^{\prime}$ is the inductive limit of the spaces $\left(\mathcal{T}_{-p}\right)_{p \in \mathcal{I}}$. We denote by $\langle\cdot, \cdot\rangle$ the dual pairings between $\mathcal{T}_{p}$ 
and $\mathcal{T}_{-p}$ and between $\mathcal{M}$ and $\mathcal{M}^{\prime}$ given by the extension of the inner product $(\cdot, \cdot)$ on $\mathcal{T}$. We preserve this notation for tensor powers of these spaces.

Additionally, we introduce the notion of symmetric tensor power of a nuclear space. The simplest way to do this is to start from usual symmetric tensor powers $\mathcal{T}_{p}^{\hat{\otimes} n}, n \in \mathbb{N}$, of Hilbert spaces. Using the definition

$$
\mathcal{M}^{\hat{\otimes} n}:=\operatorname{prlim}_{p \in \mathcal{I}} \mathcal{T}_{p}^{\hat{\otimes} n}
$$

one can prove, see e.g [Pie72] and [Sch71], that $\mathcal{M}^{\hat{\otimes} n}$ is a nuclear space which is called the $n$-th symmetric tensor power of $\mathcal{M}$. The dual space $\mathcal{M}^{i \otimes n}$ can be written as

$$
\mathcal{M}^{\prime \hat{\otimes} n}=\operatorname{indlim}_{p \in \mathcal{I}} \mathcal{T}_{-p}^{\hat{\otimes} n}
$$

All the results quoted above also hold for complex spaces.

The symmetric (or Boson) Fock space $\Gamma(\mathcal{T})$ of $\mathcal{T}$ is given by the completion of $\bigoplus_{n=0}^{\infty} \mathcal{T}_{\mathbb{C}}^{\hat{\otimes} n}\left(\mathcal{T}_{\mathbb{C}}^{\hat{\otimes} 0}:=\mathbb{C}\right)$ w.r.t. the Hilbertian norm

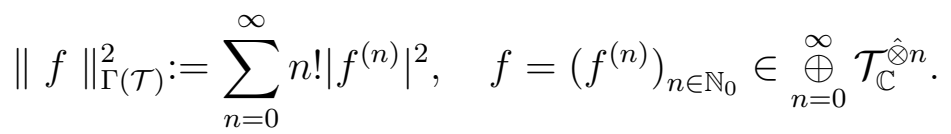

Example 2.1 (i) Let $\mathcal{T}$ be the real vector space of all hermitian HilbertSchmidt operators defined on a complex separable Hilbert space $\mathcal{H}$. Choosing an orthonormal basis $\left(e_{i}\right)_{i \in \mathbb{N}}$ of $\mathcal{H}$ we can identify a given operator $A \in \mathcal{T}$ with the matrix

$$
\left(a_{i j}\right)_{i, j \in \mathbb{N}}, \quad a_{i j}=\left(e_{i}, A e_{j}\right)_{\mathcal{H}},
$$

where $(\cdot, \cdot)_{\mathcal{H}}$ is the inner product of $\mathcal{H}$. Since the trace of the composition of two Hilbert-Schmidt operators is well-defined, on $\mathcal{T} \times \mathcal{T}$ we can introduce the bilinear form

$$
(A, B) \mapsto \operatorname{Tr}(B A) \in \mathbb{R}
$$

Since elements from $\mathcal{T}$ are hermitian Hilbert-Schmidt operators the mapping (4) is a real valued, positive definite, symmetric, bilinear form on $\mathcal{T} \times \mathcal{T}$. Hence on $\mathcal{T}$ we can define the scalar product

$$
(A, B):=\operatorname{Tr}(B A), \quad A, B \in \mathcal{T} .
$$


Now by standard methods one can verify that $(\mathcal{T},(\cdot, \cdot))$ is a real separable Hilbert space.

$\mathcal{M}$ we choose as the subspace of $\mathcal{T}$ such that each operator $F \in \mathcal{M}$ represented as in (3) has only finite entries. This space can be equiped with a topology such that it is a nuclear space with the properties required above, see BK95. Note that if $\mathcal{H}$ is not finite dimensional then $\mathcal{M}$ is not a separable nuclear space, i.e., it can not be represented as the projective limit of a countably set of Hilbert spaces.

(ii) In the finite dimensional case, i.e., $\mathcal{H}$ is an $N$ dimensional, $N \in \mathbb{N}$, complex Hilbert space, we can identify

$$
\mathcal{M}=\mathcal{T}=\mathcal{M}^{\prime} \simeq \operatorname{Mat}_{N}^{h}(\mathbb{C}),
$$

where $\operatorname{Mat}_{N}^{h}(\mathbb{C})$ is the real Hilbert space of Hermitian $(N \times N)$-matrices over the field $\mathbb{C}$.

In order to introduce a probability measure on the vector space $\mathcal{M}^{\prime}$ we consider the $\sigma$-algebra $\mathcal{C}_{\sigma}\left(\mathcal{M}^{\prime}\right)$ generated by cylinder sets

$$
C_{B_{1}, \ldots, B_{n}}^{F_{1}, \ldots, F_{n}}=\left\{A \in \mathcal{M}^{\prime} \mid\left\langle A, F_{1}\right\rangle \in B_{1}, \ldots,\left\langle A, F_{n}\right\rangle \in B_{n}\right\}
$$

where $F_{i} \in \mathcal{M}, B_{i} \in \mathcal{B}(\mathbb{R}), 1 \leq i \leq n, n \in \mathbb{N}$, and $\mathcal{B}(\mathbb{R})$ denotes the Borel $\sigma$-algebra on $\mathbb{R}$.

The standard Gaussian measure $\mu$ on $\left(\mathcal{M}^{\prime}, \mathcal{C}_{\sigma}\left(\mathcal{M}^{\prime}\right)\right)$ is given by its characteristic function

$$
C(F)=\int_{\mathcal{M}^{\prime}} \exp (i\langle A, F\rangle) d \mu(A)=\exp \left(-\frac{1}{2}|F|^{2}\right), \quad F \in \mathcal{M},
$$

via Minlos' theorem, see e.g. BK95], Hid80] and [HKPS93]. $\left(\mathcal{M}^{\prime}, \mathcal{C}_{\sigma}\left(\mathcal{M}^{\prime}\right), \mu\right)$ is the basic probability space throughout this work. The central space in our setup is the space of complex valued functions which are square-integrable with respect to this measure

$$
L^{2}(\mu):=L^{2}\left(\mathcal{M}^{\prime}, \mathcal{C}_{\sigma}\left(\mathcal{M}^{\prime}\right), \mu\right) .
$$

Consider the space $\mathcal{P}\left(\mathcal{M}^{\prime}\right) \subset L^{2}(\mu)$ of smooth polynomials on $\mathcal{M}^{\prime}$ :

$$
\mathcal{P}\left(\mathcal{M}^{\prime}\right):=\left\{\varphi \mid \varphi(A)=\sum_{n=0}^{N}\left\langle A^{\otimes n}, \tilde{\varphi}^{(n)}\right\rangle, \tilde{\varphi}^{(n)} \in \mathcal{M}_{\mathbb{C}}^{\otimes n}, A \in \mathcal{M}^{\prime}, N \in \mathbb{N}_{0}\right\} .
$$


It is well-known that any $\varphi \in \mathcal{P}\left(\mathcal{M}^{\prime}\right)$ can be written as a smooth Wick polynomial, i.e.,

$$
\mathcal{P}\left(\mathcal{M}^{\prime}\right)=\left\{\varphi \mid \varphi(A)=\sum_{n=0}^{N}\left\langle: A^{\otimes n}:, \varphi^{(n)}\right\rangle, \varphi^{(n)} \in \mathcal{M}_{\mathbb{C}}^{\hat{\otimes} n}, A \in \mathcal{M}^{\prime}, N \in \mathbb{N}_{0}\right\}
$$

and that $\mathcal{P}\left(\mathcal{M}^{\prime}\right)$ is dense in $L^{2}(\mu)$.

Smooth Wick monomials of different order are orthogonal w.r.t. the standard inner product in $L^{2}(\mu)$. Furthermore, we can construct Wick monomials with kernels $f^{(n)} \in \mathcal{T}_{\mathbb{C}}^{\hat{\otimes} n}$ in the sense of measurable functions by using an approximation. More precisely, for any sequence $\left(\varphi_{j}^{(n)}\right)_{j \in \mathbb{N}_{\mathbb{C}}} \subset \mathcal{M}_{\mathbb{C}}^{\otimes}$ which converges to $f^{(n)}$ in $\mathcal{T}_{\mathbb{C}}^{\hat{\otimes} n}$ we have that $\left(\left\langle: A^{\otimes n}:, \varphi_{j}^{(n)}\right\rangle\right)_{j \in \mathbb{N}}$ is a Cauchy sequence in $L^{p}(\mu), p \geq 1$, see e.g. [BK95]. Since the $L^{p}(\mu)$ spaces are complete the sequence $\left(\left\langle: A^{\otimes n}:, \varphi_{j}^{(n)}\right\rangle\right)_{j \in \mathbb{N}}$ converges and we use $\left\langle: A^{\otimes n}:, f^{(n)}\right\rangle$, as a formal notation for the limiting monomial. For Wick monomials associated to the kernels $f^{(n)} \in \mathcal{H}_{\mathbb{C}}^{\hat{\otimes} n}$ and $h^{(m)} \in \mathcal{T}_{\mathbb{C}}^{\hat{\otimes} m}, n, m \in \mathbb{N}_{0}$, we have the following orthogonality property:

$$
\left.\int_{\mathcal{M}^{\prime}} \overline{\left\langle: A^{\otimes n}:, f^{(n)}\right\rangle}\left\langle: A^{\otimes m}:, h^{(m)}\right\rangle d \mu(A)=\delta_{n, m} n ! \overline{\left(f^{(n)}\right.}, h^{(n)}\right)
$$

$\left(\delta_{n, m}\right.$ is the Kronecker delta).

The well-known Segal isomorphism between $L^{2}(\mu)$ and $\Gamma(\mathcal{T})$ establishes the Itô-Segal-Wiener chaos decomposition of an element $f \in L^{2}(\mu)$ :

$$
f=\sum_{n=0}^{\infty}\left\langle: A^{\otimes n}:, f^{(n)}\right\rangle, \quad f^{(n)} \in \mathcal{T}_{\mathbb{C}}^{\hat{\otimes} n}
$$

and its norm is given by

$$
\|f\|_{L^{2}(\mu)}^{2}=\sum_{n=0}^{\infty} n !\left|f^{(n)}\right| .
$$

\subsection{Generalized functions on matrix spaces}

For our considerations the space $L^{2}(\mu)$ is too small. A convenient way to solve this problem is to introduce a space of test functions in $L^{2}(\mu)$ and use its larger dual space. In Gaussian analysis there exist various triples of test 
and generalized functions with $L^{2}(\mu)$ as a central space, here we choose the Hida triple

$$
(\mathcal{M}) \subset L^{2}(\mu) \subset(\mathcal{M})^{\prime}
$$

where $(\mathcal{M})$ is the space of test functions. It is containing the space of smooth polynomials $\mathcal{P}\left(\mathcal{N}^{\prime}\right)$ and is densely and topologically embedded in $L^{2}(\mu)$. Its topologically dual $(\mathcal{M})^{\prime}$ is the space of Hida distributions, see [KS78, [Kon80], [Kon91], KT80], BK95, "PS91], "HKPS93, and [KLP+96]. Instead of reproducing the construction of this triple we give a complete and convenient characterization via the $S$-transform.

Consider the Wick exponential

$$
\begin{aligned}
: \exp (\langle A, F\rangle): & :=\exp \left(\langle A, F\rangle-\frac{1}{2}|F|^{2}\right) \\
& =\sum_{n=0}^{\infty} \frac{1}{n !}\left\langle: A^{\otimes n}:, F^{\otimes n}\right\rangle, \quad A \in \mathcal{M}^{\prime}, F \in \mathcal{M} .
\end{aligned}
$$

The $S$-transform of $\Phi \in(\mathcal{M})^{\prime}$ at $F \in \mathcal{M}$ is defined as

$$
S \Phi(F):=\langle\langle\Phi,: \exp (\langle\cdot, F\rangle):\rangle\rangle,
$$

here $\langle\langle\cdot, \cdot\rangle\rangle$ denotes the bilinear dual pairing between $(\mathcal{M})$ and $(\mathcal{M})^{\prime}$. Since $: \exp (\langle\cdot, F\rangle): \in(\mathcal{M})$ this pairing is well-defined. Formally we can write the $S$-transform as an integral

$$
S \Phi(F)=\int_{\mathcal{M}^{\prime}} \Phi(A): \exp (\langle A, F\rangle): d \mu(A), \quad \Phi \in(\mathcal{M})^{\prime} .
$$

Rigorously, this representation is valid for e.g. square-integrable functions. Furthermore, we denote by $\mathbb{E}(\Phi):=\langle\langle\Phi, 1\rangle\rangle=S \Phi(0)$ the (generalized) expectation of a Hida distribution.

In order to characterize the Hida distributions we need the definition of a $U$-functional.

Definition 2.2 Let $U: \mathcal{M} \rightarrow \mathbb{C}$ be such that:

(i) for all $F, G \in \mathcal{M}$ the mapping $l \mapsto U(G+l F)$ from $\mathbb{R}$ into $\mathbb{C}$ has an entire extension to $l \in \mathbb{C}$, and

(ii) there exist constants $K, C>0$ and a continuous quadratic form $Q$ on $\mathcal{M}$ so that for all $F \in \mathcal{M}, z \in \mathbb{C}$,

$$
|U(z F)| \leq K \exp \left(C|z|^{2} Q(F)\right) .
$$

Then $U$ is called a $U$-functional. 
This is the base of the following characterization theorem. For the proof we refer to [PS91] and [KLP+96].

Theorem 2.3 The mapping $U: \mathcal{M} \rightarrow \mathbb{C}$ is the $S$-transform of an element in $(\mathcal{M})^{\prime}$ if and only if it is a $U$-functional.

Theorem 2.3 enables us to discuss convergence of a sequence of generalized functions. The following corollary is proved in HKPS93, [PS91] and [KLP+96].

Corollary 2.4 Let $\left(U_{n}\right)_{n \in \mathbb{N}}$ denote a sequence of $U$-functionals such that (i) $\left(U_{n}(F)\right)_{n \in \mathbb{N}}$ is a Cauchy sequence for all $F \in \mathcal{M}$, and

(ii) there exists a continuous quadratic form $Q$ on $\mathcal{M}$ and $C, D>0$ such that:

$$
\left|U_{n}(z F)\right| \leq D \exp \left(C|z|^{2} Q(F)\right)
$$

for all $F \in \mathcal{M}, z \in \mathbb{C}$, and almost all $n \in \mathbb{N}$.

Then $\left(S^{-1} U_{n}\right)_{n \in \mathbb{N}}$ converges strongly in $(\mathcal{M})^{\prime}$.

Sometimes it is more convenient to use the so called $T$-transform of a Hida distribution $\Phi$. This transform is a generalization of the Fourier transform in finite dimensional analysis and is defined as

$$
T \Phi(F):=\langle\langle\Phi, \exp (i\langle\cdot, F\rangle)\rangle\rangle, \quad F \in \mathcal{M} .
$$

$S$ - and $T$-transform have entire extensions to $\mathcal{M}_{\mathbb{C}}$ and are related by the following formula

$$
T \Phi(F)=C(F) S \Phi(i F), \quad F \in \mathcal{M}_{\mathbb{C}}
$$

The theorems proved above are also valid for the $T$-transform.

\section{Symplectic Feynman distributions}

Recall the matrix model with the partition function (1) defined in the introduction.

$$
Z(N, d, g)=\int_{\mathbb{R}^{2 d N^{2}}} \exp (i S(A, B, g)) d A d B, \quad N, d \in \mathbb{N}, g \in \mathbb{R},
$$


where the action is

$$
S(A, B, g)=\operatorname{Tr}\left(A_{\mu} B_{\mu}\right)+\frac{g}{2 N} \operatorname{Tr}\left(A_{\mu} B_{\nu} A_{\mu} B_{\nu}\right), \quad A_{\mu}, B_{\mu} \in \operatorname{Mat}_{N}^{h}(\mathbb{C}) .
$$

Since the integrand in formula (5) is not integrable with respect to the Lebesgue measure on $\mathbb{R}^{2 d N^{2}}$ we have to give a rigorous meaning to the heuristic formula for the partition function.

In this section our aim is to realize the partition function for $g=0$ as the generalized expectation of a Hida distribution. Firstly we regularize the integrand in (5) and afterwards we show that if we remove the regulation we obtain a well-defined element in the space of Hida distributions.

\subsection{One dimensional case}

Let us at first consider the case $N=1$. The suitable underlying nuclear triple is

$$
\mathcal{M}=\mathcal{T}=\mathcal{M}^{\prime}=\operatorname{Mat}_{1,2 d}^{h}(\mathbb{C}) \simeq \mathbb{R}^{2 d},
$$

where $\operatorname{Mat}_{1,2 d}^{h}(\mathbb{C}):=\left(\operatorname{Mat}_{1}^{h}(\mathbb{C})\right)^{2 d}$ is the real vector space of $2 d$-vectors having in each component a hermitian $1 \times 1$-matrices, see Example 2.1(i), (ii). This space is isomorphic to $\mathbb{R}^{2 d}$. The corresponding Gaussian measure we denote by $\mu_{1, d}$; using the isomorphism to $\mathbb{R}^{2 d}$ the measure $\mu_{1, d}$ is just the standard Gaussian measure on $\mathbb{R}^{2 d}$.

Let us define the regularized symplectic Feynman distribution

$$
\Phi_{1, d, \epsilon}(a, b):=(2 \pi)^{d} \exp \left(-\frac{1}{2}\left(\left(\begin{array}{l}
a \\
b
\end{array}\right), K_{\epsilon}\left(\begin{array}{l}
a \\
b
\end{array}\right)\right)_{\mathbb{R}^{2 d}}\right), \quad\left(\begin{array}{l}
a \\
b
\end{array}\right) \in \mathbb{R}^{2 d},
$$

where $a, b \in \mathbb{R}^{d}$ and $(\cdot, \cdot)_{\mathbb{R}^{2 d}}$ is the standard scalar product in $\mathbb{R}^{2 d}$. The operator $K_{\epsilon}$ is defined as

$$
K_{\epsilon}=-\left((1-\epsilon) I d_{\mathbb{R}^{2 d}}+i\left(\begin{array}{cc}
0 & I d_{\mathbb{R}^{d}} \\
I d_{\mathbb{R}^{d}} & 0
\end{array}\right)\right), \quad \epsilon>0 .
$$

Obviously, $\Phi_{1, d, \epsilon} \in L^{1}\left(\mu_{1, d}\right)$ and its expectation is given by

$$
\mathbb{E}_{\mu_{1, d}}\left(\Phi_{1, d, \epsilon}\right)=\int_{\mathbb{R}^{2 d}} \exp \left(-\frac{\epsilon}{2}\left(a_{\mu} a_{\mu}+b_{\mu} b_{\mu}\right)+i a_{\mu} b_{\mu}\right) d a d b .
$$


We are interested in the limit $\epsilon \rightarrow 0$ of $\Phi_{1, d, \epsilon}$. There is no hope to find this limit in $L^{1}\left(\mu_{1, d}\right)$. Since for $\epsilon$ small enough the regularized symplectic Feynman distribution $\Phi_{1, d, \epsilon}$ are not elements from $L^{2}\left(\mu_{1, d}\right)$ it is even a priori not clear if they are Hida distributions.

Lemma 3.1 The regularized symplectic Feynman distributions $\Phi_{1, d, \epsilon}, \epsilon>0$, are Hida distributions.

Proof: Since $\Phi_{1, d, \epsilon} \in L^{1}\left(\mu_{1, d}\right)$ we can take its $T$-transform at $(f, g) \in \mathbb{R}^{2 d}$ :

$$
\begin{aligned}
T \Phi_{1, d, \epsilon}(a, b) & =(2 \pi)^{d} \int_{\mathbb{R}^{2 d}} \exp \left(i\left(a_{\mu} f_{\mu}+b_{\mu} g_{\mu}\right)\right) \Phi_{1, d, \epsilon}(a, b) d \mu_{1, d}(a, b) \\
& =\left(\frac{2 \pi}{\sqrt{\epsilon^{2}+1}}\right)^{d} \exp \left(-\frac{1}{2}\left(\left(\begin{array}{c}
f \\
g
\end{array}\right),\left(K_{\epsilon}+I d_{\mathbb{R}^{2 d}}\right)^{-1}\left(\begin{array}{l}
f \\
g
\end{array}\right)\right)_{\mathbb{R}^{2 d}}\right) \\
& =\left(\frac{2 \pi}{\sqrt{\epsilon^{2}+1}}\right)^{d} \exp \left(-\frac{\epsilon\left(f_{\mu} f_{\mu}+g_{\mu} g_{\mu}\right)+2 i f_{\mu} g_{\mu}}{2\left(\epsilon^{2}+1\right)}\right) .
\end{aligned}
$$

It is easy to show that $T \Phi_{1, d, \epsilon}$ is a $U$-functional. Thus, an application of Theorem 2.3 gives us that $\Phi_{1, d, \epsilon} \in\left(\text { Mat }_{1,2 d}^{h}\right)^{\prime}$.

Next we prove that $\Phi_{1, d, \epsilon}$ converges in $\left(M a t_{1,2 d}^{h}\right)^{\prime}$ as $\epsilon \rightarrow 0$.

Proposition 3.2 There exists a Hida distribution $\Phi_{1, d}$ such that

$$
\lim _{\epsilon \rightarrow 0} \Phi_{1, d, \epsilon}=\Phi_{1, d}
$$

in the strong sense. The T-transform of $\Phi_{1, d}$ is given by

$$
T \Phi_{1, d}(a, b)=(2 \pi)^{d} \exp \left(-i f_{\mu} g_{\mu}\right), \quad(a, b) \in \mathbb{R}^{2 d} .
$$

Proof: Let us choose a sequence $\left(\epsilon_{m}\right)_{m \in \mathbb{N}}$ which converges to 0 . Lemma 3.1 tells us that $\left(T \Phi_{1, d, \epsilon_{m}}\right)_{m \in \mathbb{N}}$ is a convergent sequence of $U$-functionals. Furthermore, there exists an $M \in \mathbb{N}$ such that

$$
\begin{aligned}
\left|T \Phi_{1, d, \epsilon_{m}}(z(f, g))\right| & \leq\left(\frac{2 \pi}{\sqrt{\epsilon_{m}^{2}+1}}\right)^{d} \exp \left(|z|^{2}\left|\frac{\epsilon_{m}\left(f_{\mu} f_{\mu}+g_{\mu} g_{\mu}\right)+2 i f_{\mu} g_{\mu}}{2\left(\epsilon_{m}^{2}+1\right)}\right|\right) \\
& \leq(2 \pi)^{d} \exp \left(2|z|^{2}\left|\left(\begin{array}{c}
f \\
g
\end{array}\right)\right|_{\mathbb{R}^{2 d}}^{2}\right), \quad \forall m \geq M, z \in \mathbb{C} .
\end{aligned}
$$

Therefore, the sequence $\left(T \Phi_{1, d, \epsilon_{m}}\right)_{m \in \mathbb{N}}$ fulfills the properties required in Corollary 2.4. Consequently, $\left(T \Phi_{1, d, \epsilon_{m}}\right)_{m \in \mathbb{N}}$ converges strongly in $\left(M a t_{1,2 d}^{h}\right)^{\prime}$. The limiting function we denote by $\Phi_{1, d}$ and since strong convergence implies weak convergence the $T$-transform of $\Phi_{1, d}$ is given by (17). 


\subsection{Finite dimensional case}

Now we generalize these considerations to arbitrary $N \in \mathbb{N}$. For this the appropriate basic nuclear triple is

$$
\mathcal{M}=\mathcal{T}=\mathcal{M}^{\prime}=\operatorname{Mat}_{N, 2 d}^{h}(\mathbb{C})
$$

and we denote by $\mu_{N, d}$ the standard Gaussian measure on this space. For the computation of integrals w.r.t. $\mu_{N, d}$ it is convenient to have an complete orthogonal system in $M a t_{N, d}^{h}$. This enables the factorization of $\mu_{N, d}$ into a product of Gaussian measures on $\mathbb{R}$. In $\operatorname{Mat}_{N}^{h}(\mathbb{C})$ equiped with the inner product given by the trace we consider the complete orthogonal system

$$
C O S_{n}=\left\{e_{k, l}, 0 \leq k \leq l \leq N\right\} \cup\left\{i J e_{k, l}, 0 \leq k<l \leq N\right\}
$$

where $e_{k, k}^{r s}=1$ if $r=s=k$ and 0 otherwise, and for $k \neq l$ is $e_{k, l}^{r s}=1 / 2$ if $(r, s)=(k, l)$ or $(r, s)=(l, k)$ and 0 otherwise. The map $J$ is given by multiplication with the symplectic matrix

$$
J=\left(\begin{array}{cc}
0 & I d_{\mathbb{R}^{N}} \\
-I d_{\mathbb{R}^{N}} & 0
\end{array}\right)
$$

$\left(I d_{\mathcal{H}}\right.$ denotes the identity operator on the Hilbert space $\left.\mathcal{H}\right)$. Note that

$$
\begin{aligned}
\Re A^{k l} & =\operatorname{Tr}\left(A e_{k, l}\right), \quad 0 \leq k \leq l \leq N, \quad A \in \operatorname{Mat}_{N}^{h}(\mathbb{C}), \\
\Im A^{k l} & =\operatorname{Tr}\left(A i J e_{k, l}\right), \quad 0 \leq k<l \leq N,
\end{aligned}
$$

and that the system $C O S_{N}$ is not normalized. By standard methods the system $C O S_{N}$ can be extended to an orthogonal basis $C O S_{N, d}$ of the product space $M a t_{N, d}^{h}(\mathbb{C})$. The $d$-vector in $C O S_{N, d}$ having in the $\mu$-th component the matrix $e_{k, l}$ or $J e_{k, l}$ and the zero matrix in the other components we denote by $e_{\mu, k, l}$ or $J e_{\mu, k, l}$, respectively, $1 \leq \mu \leq d, 1 \leq k \leq l \leq N$.

In this setting we define the regularized symplectic Feynman distribution

$\Phi_{N, d, \epsilon}:=2^{d N} \pi^{d N^{2}} \exp \left(-\frac{1}{2}\left(\left(\begin{array}{l}A \\ B\end{array}\right), K_{\epsilon}\left(\begin{array}{l}A \\ B\end{array}\right)\right)_{M a t_{N, 2 d}^{h}}\right),\left(\begin{array}{l}A \\ B\end{array}\right) \in M a t_{N, 2 d}^{h}$,

where $A, B \in M a t_{N, d}^{h}$ and $(\cdot, \cdot)_{M a t_{N, 2 d}^{h}}$ is the scalar product on $M a t_{N, 2 d}^{h}$ given by the trace, see Example 2.1(i), (ii) (since there is no danger of confusion 
we denote from now on $M a t_{N, 2 d}^{h}(\mathbb{C})$ by $\left.M a t_{N, 2 d}^{h}\right)$. The operator $K_{\epsilon}$ is defined as

$$
K_{\epsilon}=-\left((1-\epsilon) I d_{M a t_{N, 2 d}^{h}}+i\left(\begin{array}{cc}
0 & I d_{M a t_{N, d}^{h}}^{h} \\
I d_{M a t_{N, d}^{h}} & 0
\end{array}\right)\right), \quad \epsilon>0 .
$$

As in the case $N=1$ we have $\Phi_{N, d, \epsilon} \in L^{1}\left(\mu_{N, d}\right)$ and its expectation is given by

$$
\mathbb{E}_{\mu_{N, d}}\left(\Phi_{N, d, \epsilon}\right)=\int_{\mathbb{R}^{2 d N^{2}}} \exp \left(-\frac{\epsilon}{2} \operatorname{Tr}\left(A_{\mu} A_{\mu}+B_{\mu} B_{\mu}\right)+i \operatorname{Tr}\left(A_{\mu} B_{\mu}\right) d A d B\right.
$$

Lemma 3.3 The regularized symplectic Feynman distributions $\Phi_{N, d, \epsilon}, \epsilon>0$, are Hida distributions.

Proof: We use the basis $C O S_{N, d}$ of $M a t_{N, d}^{h}$ in order to evaluation the $T$ transform of $\Phi_{N, d, \epsilon}$. Doing this, the corresponding integral factories into a product of Gaussian measures on $\mathbb{R}$ and we obtain

$$
\begin{aligned}
& T \Phi_{N, d, \epsilon}(F, G) \\
& =2^{d N}\left(\frac{\pi}{\sqrt{\epsilon^{2}+1}}\right)^{d N^{2}} \exp \left(-\frac{\epsilon \operatorname{Tr}\left(F_{\mu} F_{\mu}+G_{\mu} G_{\mu}\right)+2 i \operatorname{Tr}\left(F_{\mu} G_{\mu}\right)}{2\left(\epsilon^{2}+1\right)}\right),
\end{aligned}
$$

$F, G \in M a t_{N, d}^{h}$. Obviously, $T \Phi_{N, d, \epsilon}$ is a $U$-functional and therefore $\Phi_{N, d, \epsilon} \in$ $\left(M_{a, 2 d}^{h}\right)^{\prime}$ by characterization.

Proposition 3.4 There exists a Hida distribution $\Phi_{N, d}$ such that

$$
\lim _{\epsilon \rightarrow 0} \Phi_{N, d, \epsilon}=\Phi_{N, d}
$$

in the strong sense. The T-transform of $\Phi_{N, d}$ is given by

$$
T \Phi_{N, d}(F, G)=2^{d N} \pi^{d N^{2}} \exp \left(-i \operatorname{Tr}\left(F_{\mu} G_{\mu}\right)\right), \quad(F, G) \in M a t_{N, 2 d}^{h} .
$$

Proof: The proof of this proposition is an easy generalization of that of Proposition 3.2. 
Remark 3.5 (i) Now we can realize the partition functions $Z(N, d, 0)$ as the generalized expectation of $\Phi_{N, d}$, i.e.,

$$
Z(N, d, 0):=\mathbb{E}_{\mu_{N, d}}\left(\Phi_{N, d}\right), \quad \Phi_{N, d} \in\left(M a t_{N, 2 d}^{h}\right)^{\prime} .
$$

(ii) Observe, that the sequence $\left(\Phi_{N, d}\right)_{N \in \mathbb{N}}$ can not be found in one space of Hida distributions. For different $N \in \mathbb{N}$ they are elements of different spaces of Hida distributions for which a priori there exists no relation. Since we are interested in the limit $N \rightarrow \infty$ next we construct a space of Hida distributions which contains all the $\Phi_{N, d}, N \in \mathbb{N}$ (up to a natural identification).

\subsection{Infinite dimensional case}

Recall the nuclear space of finite rank matrices discussed in Example 2.1(i). We denote by $\mathcal{M}^{2 d}$ the nuclear space which is $2 d$-times the product of this space with itself. In the same way we construct the product space $\mathcal{T}^{2 d}$ and obtain the nuclear triple

$$
\mathcal{M}^{2 d} \subset \mathcal{T}^{2 d} \subset \mathcal{M}^{\prime 2 d}
$$

The associated standard Gaussian measure we denote by $\mu_{d}$. As described in Example 2.1(i) we can represent the spaces (9) as matrix spaces. Using this representation we define the mapping

$$
P_{N}: \mathcal{M}^{\prime d} \rightarrow \mathcal{M}^{d}
$$

as the projection onto the subspace of $\mathcal{M}^{d}$ generated by $C O S_{N, d}$ (naturally embedded into $\left.\mathcal{M}^{d}\right)$. The systems $\left(C O S_{N, d}\right)_{N \in \mathbb{N}}$ can not only be naturally embedded into $\mathcal{M}^{d}$, they also can be used to constructed an orthogonal basis of $\mathcal{T}^{d}$ which we denote by $C O S_{d}$. We define

$$
\tilde{\Phi}_{N, d, \epsilon}:=2^{d N} \pi^{d N^{2}} \exp \left(-\frac{1}{2}\left(\left(\begin{array}{c}
P_{N} A \\
P_{N} B
\end{array}\right), K_{\epsilon}\left(\begin{array}{l}
P_{N} A \\
P_{N} B
\end{array}\right)\right)_{\mathcal{T}^{2 d}}\right),\left(\begin{array}{l}
A \\
B
\end{array}\right) \in \mathcal{M}^{\prime 2 d}
$$

where $A, B \in \mathcal{M}^{\prime d}$ and $(\cdot, \cdot)_{\mathcal{T}^{2 d}}$ is the scalar product on $\mathcal{T}^{2 d}$ given by the trace, see Example 2.1(i). The operator $K_{\epsilon}$ is defined as

$$
K_{\epsilon}=-\left((1-\epsilon) I d_{\mathcal{M}^{\prime 2 d}}+i\left(\begin{array}{cc}
0 & I d_{\mathcal{M}^{\prime d}} \\
I d_{\mathcal{M}^{\prime d}} & 0
\end{array}\right)\right), \quad \epsilon>0 .
$$


The regularized symplectic Feynman distributions $\tilde{\Phi}_{N, d, \epsilon}$ and $\Phi_{N, d, \epsilon}$ are essentially the same. They are only defined on different spaces and we have

$$
\begin{aligned}
\mathbb{E}_{\mu_{d}}\left(\tilde{\Phi}_{N, d, \epsilon}\right) & =\int_{\mathbb{R}^{2 d N^{2}}} \exp \left(-\frac{\epsilon}{2} \operatorname{Tr}\left(A_{\mu} A_{\mu}+B_{\mu} B_{\mu}\right)+i \operatorname{Tr}\left(A_{\mu} B_{\mu}\right) d A d B\right. \\
& =\mathbb{E}_{\mu_{N, d}}\left(\Phi_{N, d, \epsilon}\right) .
\end{aligned}
$$

Hence, from now on we identify $\tilde{\Phi}_{N, d, \epsilon}$ with $\Phi_{N, d, \epsilon}$.

Theorem 3.6 There exists a Hida distribution $\Phi_{d} \in\left(\mathcal{M}^{2 d}\right)^{\prime}$ such that

$$
\lim _{N \rightarrow \infty} \lim _{\epsilon \rightarrow 0} R^{-1} \Phi_{N, d, \epsilon}=\lim _{\epsilon \rightarrow 0} \lim _{N \rightarrow \infty} R^{-1} \Phi_{N, d, \epsilon}=\Phi_{d}, R=2^{d N}\left(\frac{\pi}{\sqrt{\epsilon^{2}+1}}\right)^{d N^{2}},
$$

in the strong sense. The T-transform of $\Phi_{d}$ is given by

$$
T \Phi_{d}(F, G)=\exp \left(-i \operatorname{Tr}\left(F_{\mu} G_{\mu}\right)\right), \quad(F, G) \in \mathcal{M}^{2 d} .
$$

Proof: Let us choose a sequence $\left(\epsilon_{m}\right)_{m \in \mathbb{N}}$ which converges to 0 . The $T$ transform of $N^{-1} \Phi_{N, d, \epsilon_{m}}$ at $(F, G) \in \mathcal{M}^{2 d}$ is

$$
\begin{aligned}
& R^{-1} T \Phi_{N, d, \epsilon_{m}}(F, G) \\
= & \int_{\mathcal{M}^{\prime 2 d}} \exp \left(i\left(A_{\mu} F_{\mu}+B_{\mu} G_{\mu}\right)\right) \Phi_{N, d, \epsilon_{m}}(A, B) d \mu_{d}(A, B) \\
= & \exp \left(-\frac{1}{2}\left(\left(\begin{array}{l}
P_{N} F \\
P_{N} G
\end{array}\right),\left(K_{\epsilon_{m}}+I d_{\mathcal{M}^{\prime 2 d}}\right)^{-1}\left(\begin{array}{l}
P_{N} F \\
P_{N} G
\end{array}\right)\right)_{\mathcal{T}^{2 d}}\right) \\
\cdot & \exp \left(-\frac{1}{2}\left(\left(\begin{array}{l}
P_{N}^{c} F \\
P_{N}^{c} G
\end{array}\right),\left(\begin{array}{l}
P_{N}^{c} F \\
P_{N}^{c} G
\end{array}\right)\right)_{\mathcal{T}^{2 d}}\right),
\end{aligned}
$$

where $P_{N}^{c}:=I d_{\mathcal{M}^{\prime d}}-P_{N}$. The term (11), up to the normalization, coincides essentially with the $T$-transform in the finite dimensional setting, see (8). Utilizing the same ideas as in the proof of Proposition 3.2 we have for all $(F, G) \in \mathcal{M}^{2 d}$

$$
R^{-1}\left|T \Phi_{N, d, \epsilon_{m}}(z(F, G))\right| \leq \exp \left(2|z|^{2}\left|\left(\begin{array}{l}
F \\
G
\end{array}\right)\right|_{\mathcal{T}^{2 d}}^{2}\right), \quad \forall m \geq M, z \in \mathbb{C},
$$

if we choose $M \in \mathbb{N}$ large enough. From (11]) together with (8) we can conclude that for all $(F, G) \in \mathcal{M}^{2 d}$

$$
\begin{aligned}
& \lim _{N \rightarrow \infty} \lim _{m \rightarrow \infty} R^{-1} T \Phi_{N, d, \epsilon}(F, G) \\
= & \lim _{m \rightarrow \infty} \lim _{N \rightarrow \infty} R^{-1} T \Phi_{N, d, \epsilon}(F, G)=\exp \left(-i \operatorname{Tr}\left(F_{\mu} G_{\mu}\right)\right) .
\end{aligned}
$$


Of course, the quadratic form $Q=|\cdot|_{\mathcal{T}^{2 d}}^{2}$ is continuous on $\mathcal{M}^{2 d}$. Thus, the estimate (12) together with (13) and Corollary 2.4 proves the theorem.

Corollary 3.7 There exists a Hida distribution $\Phi_{N, d} \in\left(\mathcal{M}^{2 d}\right)^{\prime}$ such that

$$
\lim _{\epsilon \rightarrow 0} \Phi_{N, d, \epsilon}=\Phi_{N, d}
$$

in the strong sense. The T-transform of $\Phi_{N, d}$ is given by

$$
\begin{aligned}
T \Phi_{N, d}(F, G)= & 2^{d N} \pi^{d N^{2}} \exp \left(-i T r\left(P_{N} F_{\mu} P_{N} G_{\mu}\right)\right) \\
\cdot & \exp \left(-\frac{1}{2}\left(\left(\begin{array}{c}
P_{N}^{c} F \\
P_{N}^{c} G
\end{array}\right),\left(\begin{array}{c}
P_{N}^{c} F \\
P_{N}^{c} G
\end{array}\right)\right)_{\mathcal{T}^{2 d}}\right), \quad(F, G) \in \mathcal{M}^{2 d} .
\end{aligned}
$$

Proof: The proof is an immediate consequence of Theorem 3.6.

Remark 3.8 (i) As in the finite dimensional case, see Remark 3.5(i), we can realize the partition function $Z(N, d, 0)$ as the generalized expectation of $\Phi_{N, d}$, i.e.,

$$
Z(N, d, 0)=\mathbb{E}_{\mu_{d}}\left(\Phi_{N, d}\right), \quad \Phi_{N, d} \in\left(\mathcal{M}^{2 d}\right)^{\prime}
$$

(ii) For test functions $(F, G) \in \mathcal{M}^{2 d}$ such that $\left(P_{N} F, P_{N} G\right)=(F, G)$ the $T$-transform of $\Phi_{N, d}$ and $2^{d N} \pi^{d N^{2}} \Phi_{d}$ coincide.

\section{Proof of Theorem}

\subsection{Knots}

In order to discuss the proof of Theorem 1.1 let us remind that a knot is a smooth embedding of an oriented circle in an oriented 3-space $\mathbb{R}^{3}$. A collection of $l$ pairwise disjoint knots is called a $l$-link. Two knots are equivalent (have the same isotopy type) if they are equivalent under a homeomorphism of $\mathbb{R}^{3}$.

A knot $K$ can be represented by a regular projection $\tilde{K}$ onto the plane having at most a finite number of transverse double points. For the plane curve $\tilde{K}$ one has to indicate which line is up $(+)$ and which line is down $(-)$ in an intersection point, see Figure 11. 

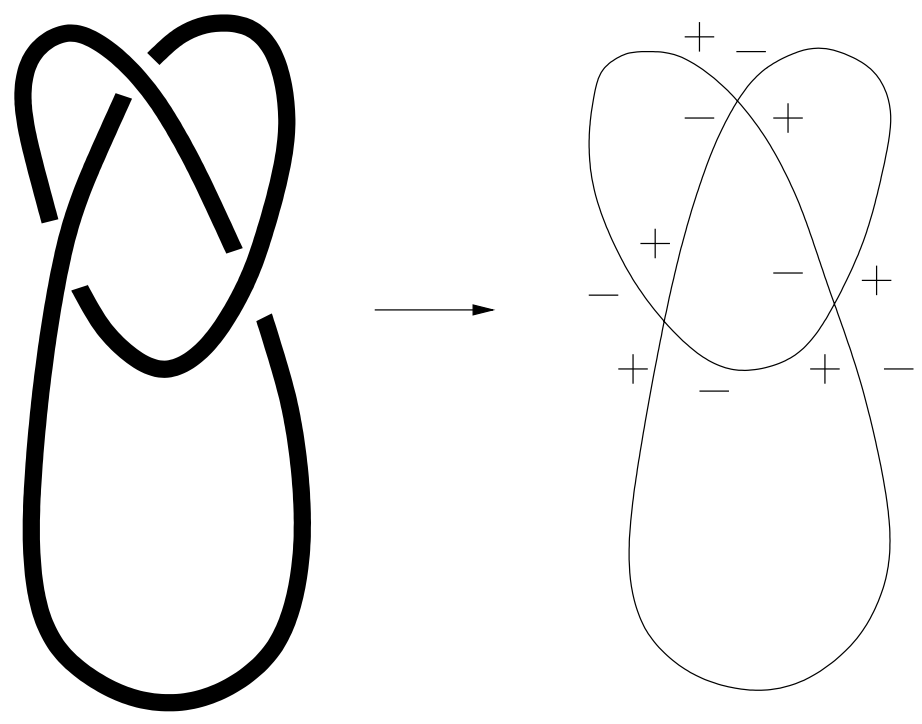

Figure 1: Trefoil
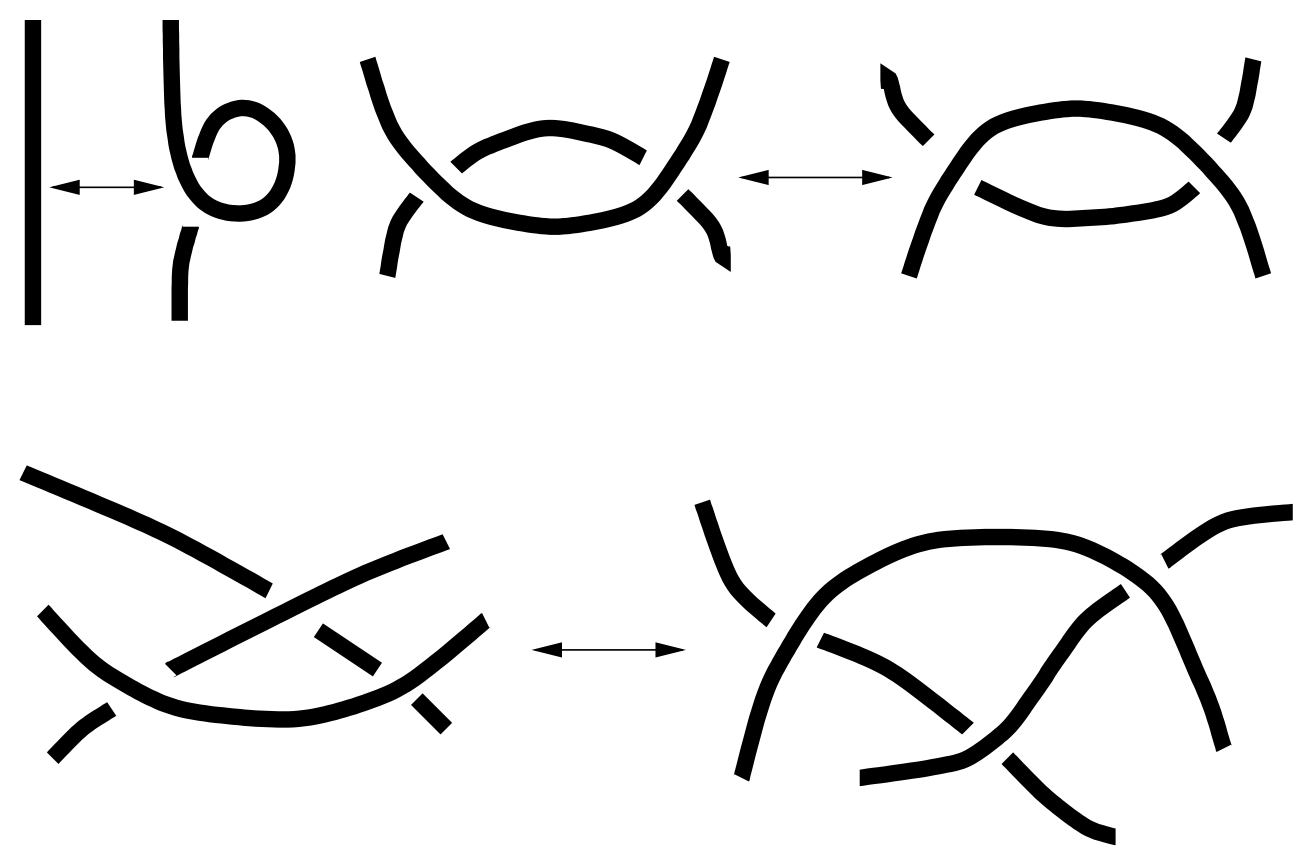

Figure 2: Reidemeister moves 
In this way we get a graph on the plane which has 4 legs in each vertex and also has the $(+-)$ prescription. This graph is called the knot diagram. A knot diagram is called alternating if it has alternating $(+)$ and $(-)$ along a line. Two knot diagrams are called Reidemeister equivalent if they define equivalent knots. Reidemeister equivalence is generated by the three moves that are illustrated in Figure 2.

\subsection{Wick theorem and propagators}

Now let us consider the integral (5). In order to prove Theorem 1.1, in AV98 the authors have expanded the partition function $Z$ into the formal perturbation series over the coupling constant $g$

$$
Z=\sum_{k=0}^{\infty} \frac{1}{k !}\left(\frac{i g}{N}\right)^{k} \int_{\mathbb{R}^{2 d N^{2}}}\left(\operatorname{Tr}\left(A_{\mu} B_{\nu} A_{\mu} B_{\nu}\right)\right)^{k} \exp \left(i \operatorname{Tr}\left(A_{\mu} B_{\mu}\right)\right) d A d B
$$

The integrals in (14) we can write as

$$
\begin{aligned}
& \left(\frac{i g}{N}\right)^{k} \int_{\mathbb{R}^{2 d N^{2}}}\left(\operatorname{Tr}\left(A_{\mu} B_{\nu} A_{\mu} B_{\nu}\right)\right)^{k} \exp \left(i \operatorname{Tr}\left(A_{\mu} B_{\mu}\right)\right) d A d B \\
= & \sum_{\mu, \nu, \alpha, \beta=1}^{d} \sum_{q_{1}, i_{1}, j_{1}, l_{1}, m_{1}, n_{1}, o_{1}, p_{1}=1}^{N} \ldots \sum_{q_{k}, i_{k}, j_{k}, l_{k}, m_{k}, n_{k}, o_{k}, p_{k}=1}^{N} \\
\cdot & \prod_{s=1}^{k}\left(\frac{i g}{N} \delta_{\mu \alpha} \delta_{\nu \beta} \delta^{i_{s} j_{s}} \delta^{l_{s} m_{s}} \delta^{n_{s} o_{s}} \delta^{p_{s} q_{s}}\right) \\
\cdot & \int_{\mathbb{R}^{2 d N^{2}}} \prod_{s=1}^{k}\left(A_{\mu}^{q_{s} i_{s}} B_{\nu}^{j_{s} l_{s}} A_{\alpha}^{m_{s} n_{s}} B_{\beta}^{o_{s} p_{s}}\right) \exp \left(i \operatorname{Tr}\left(A_{\mu} B_{\mu}\right)\right) d A d B
\end{aligned}
$$

$\left(\delta_{i, j}\right.$ and $\delta^{k, l}$ are Kronecker deltas). Our contribution to the proof of Theorem 1.1 is to give a mathematically rigorous meaning to the Gaussian integrals in (18), to prove a Wick theorem for these integrals, and to derive explicit formulas for the propagators, i.e., for the second moments of the Gaussian integrals which we obtain in the Wick theorem.

From now on we assume as underlying co-nuclear space $\mathcal{M}^{\prime 2 d}$. An elementary calculation gives

$$
\Re A_{\mu}^{k l}=\left\langle\left(\begin{array}{c}
A \\
B
\end{array}\right),\left(\begin{array}{c}
e_{\mu, k, l} \\
0
\end{array}\right)\right\rangle, \quad \Re B_{\mu}^{k l}=\left\langle\left(\begin{array}{c}
A \\
B
\end{array}\right),\left(\begin{array}{c}
0 \\
e_{\mu, k, l}
\end{array}\right)\right\rangle, \quad k \leq l,
$$




$$
\Im A_{\mu}^{k l}=\left\langle\left(\begin{array}{l}
A \\
B
\end{array}\right),\left(\begin{array}{c}
i J e_{\mu, k, l} \\
0
\end{array}\right)\right\rangle, \quad \Im B_{\mu}^{k l}=\left\langle\left(\begin{array}{l}
A \\
B
\end{array}\right),\left(\begin{array}{c}
0 \\
i J e_{\mu, k, l}
\end{array}\right)\right\rangle, \quad k<l,
$$

for $A, B \in \mathcal{M}^{\prime d}$ and $e_{\mu, k, l}, i J e_{\mu, k, l} \in C O S_{d}$, where $1 \leq \mu, \nu \leq d$ and $k, l \in \mathbb{N}$. Recall that $\Re A_{\mu}^{k l}=\Re A_{\mu}^{l k}, \Im A_{\mu}^{k l}=-\Im A_{\mu}^{l k}$ and $\Im A_{\mu}^{k k}=0$ (here we assume $A, B \in \mathcal{M}^{\prime d}$ in matrix representation as described in Example 2.1(i) and $C O S_{d}$ naturally embedded in this representation of $\left.\mathcal{M}^{\prime d}\right)$. This shows that the matrix coefficients and powers of them are elements from $\mathcal{P}\left(\mathcal{M}^{2 d}\right) \subset$ $\left(\mathcal{M}^{2 d}\right)$, see Section 2.2 .

Now let us give a mathematically rigorous definition of the integral in (18) suggested by Remark 3.8(i).

Definition 4.1 Let $N, d, k \in \mathbb{N}$ and $\Phi_{N, d}$ as in Corollary 3.7. Then we define the integral in (18) by

$$
\begin{aligned}
& \int_{\mathbb{R}^{2 d N^{2}}} \prod_{s=1}^{k} A_{\mu}^{q_{s} i_{s}} B_{\nu}^{j_{s} l_{s}} A_{\alpha}^{m_{s} n_{s}} B_{\beta}^{o_{s} p_{s}} \exp \left(i \operatorname{Tr}\left(A_{\mu} B_{\mu}\right)\right) d A d B \\
:= & \left\langle\left\langle\Phi_{N, d}, \prod_{s=1}^{k} A_{\mu}^{q_{s} i_{s}} B_{\nu}^{j_{s} l_{s}} A_{\alpha}^{m_{s} n_{s}} B_{\beta}^{o_{s} p_{s}}\right\rangle\right\rangle .
\end{aligned}
$$

Remark 4.2 Consider the formal perturbation series for the partition function $Z$ in (14). Using this series, formula (15)-(18), and Definition 4.1, we can give a mathematically rigorous definition of the partition function $Z(N, d, g)$ as a formal power series in the coupling constant $g \in \mathbb{R}$.

At a first glance it seems to be a quite elaborate task to evaluate the integral in Definition 4.1. This can be simplified be the following Wick theorem.

Theorem 4.3 (Wick theorem) Let $F_{1}, \ldots, F_{2 m}, m \in \mathbb{N}$, be elements from the matrix space $\mathcal{M}^{2 d}$ then

$$
\begin{aligned}
& \left\langle\left\langle\Phi_{d},\left\langle\cdot, F_{1}\right\rangle \cdot \ldots \cdot\left\langle\cdot, F_{2 m}\right\rangle\right\rangle\right\rangle \\
= & \sum_{\text {pairings }}\left\langle\left\langle\Phi_{d},\left\langle\cdot, F_{k_{1}}\right\rangle\left\langle\cdot, F_{l_{1}}\right\rangle\right\rangle\right\rangle \cdot \ldots \cdot\left\langle\left\langle\Phi_{d},\left\langle\cdot, F_{k_{m}}\right\rangle\left\langle\cdot, F_{l_{m}}\right\rangle\right\rangle\right\rangle,
\end{aligned}
$$

where $\sum_{\text {pairings }}$ means the sum over all $(2 m) ! /\left(2^{m} m !\right)$ ways of writing $1, \ldots$, $2 m$ as $m$ distinct (unordered) pairs $\left(k_{1}, l_{1}\right), \ldots,\left(k_{m}, l_{m}\right)$. 
Proof: It is easy to show that

$$
-\left.i \frac{d}{d t} \exp (i t\langle\cdot, F\rangle)\right|_{t=0}=\langle\cdot, F\rangle, \quad F \in \mathcal{M}^{2 d}
$$

w.r.t. the topology of $\left(\mathcal{M}^{2 d}\right)$. Since $\left(\mathcal{M}^{2 d}\right)$ is an algebra under multiplication and this multiplication is continuous we can define the point-wise product $\Phi_{d} \cdot\langle\cdot, F\rangle \in\left(\mathcal{M}^{2 d}\right)^{\prime}$ of the distribution $\Phi_{d} \in\left(\mathcal{M}^{2 d}\right)^{\prime}$ with the test function $\langle\cdot, F\rangle \in\left(\mathcal{M}^{2 d}\right)$ via the dual paring. Utilizing this product we find by an induction argument

$$
\begin{aligned}
& \left\langle\left\langle\Phi_{d},\left\langle\cdot, F_{1}\right\rangle \cdot \ldots \cdot\left\langle\cdot, F_{2 m}\right\rangle\right\rangle\right\rangle \\
= & \left.(-1)^{m} \frac{\partial^{2 m}}{\partial t_{1} \ldots \partial t_{2 m}} T \Phi_{d}\left(t_{1} F_{1}+\ldots+t_{2 m} F_{2 m}\right)\right|_{t_{1}=\ldots=t_{2 m}=0}
\end{aligned}
$$

Now under use of the explicit formula of $T \Phi_{d}$, see (10), the Wick theorem can be proved in the way as for the standard Gaussian measure (the essential feature of the $T \Phi_{d}$ is that it is an exponential of a quadratic form).

Remark 4.4 Since the indices of the matrices $A$ and $B$ in Definition 4.1 are less or equal to $N$ we can identify

$$
\left\langle\left\langle\Phi_{N, d}, \prod_{s=1}^{k} A_{\mu}^{q_{s} i_{s}} B_{\nu}^{j_{s} l_{s}} A_{\alpha}^{m_{s} n_{s}} B_{\beta}^{o_{s} p_{s}}\right\rangle\right\rangle=2^{d N} \pi^{d N^{2}}\left\langle\left\langle\Phi_{d}, \prod_{s=1}^{k} A_{\mu}^{q_{s} i_{s}} B_{\nu}^{j_{s} l_{s}} A_{\alpha}^{m_{s} n_{s}} B_{\beta}^{o_{s} p_{s}}\right\rangle\right\rangle .
$$

This is an easy consequence of formula (20) and Remark 3.8(ii).

Now the evaluation of the integrals in Definition 4.1 reduces to determination of the propagators

$$
<A_{\mu}^{k l} B_{\nu}^{m n}>:=\left\langle\left\langle\Phi_{d}, A_{\mu}^{k l} B_{\nu}^{m n}\right\rangle\right\rangle
$$

On the formal level can interpret

$$
<A_{\mu}^{k l} B_{\nu}^{m n}>=\frac{\int A_{\mu}^{k l} B_{\nu}^{m n} \exp \left(i \operatorname{Tr}\left(A_{\mu} B_{\mu}\right)\right) d A d B}{\int \exp \left(i \operatorname{Tr}\left(A_{\mu} B_{\mu}\right)\right) d A d B} .
$$

Analogously, we can define $<A_{\mu}^{k l} A_{\nu}^{m n}>$ and $<B_{\mu}^{k l} B_{\nu}^{m n}>$. 
Theorem 4.5 For the propagators we have the following identities:

$$
<A_{\mu}^{k l} B_{\nu}^{m n}>=i \delta_{\mu \nu} \delta^{k n} \delta^{l m}, \quad<A_{\mu}^{k l} A_{\nu}^{m n}>=<B_{\mu}^{k l} B_{\nu}^{m n}>=0 .
$$

Proof: Let $k<l$ and $m>N$. In the proof of Theorem 4.3 we already derived the following formula:

$$
\begin{aligned}
<A_{\mu}^{k l} B_{\nu}^{m n}> & =-\left.\frac{\partial^{2}}{\partial t_{1} \partial t_{2}} T \Phi_{d}\left(t_{1}\left(\begin{array}{c}
e_{\mu, k, l} \\
0
\end{array}\right)+t_{2}\left(\begin{array}{c}
0 \\
e_{\nu, n, m}
\end{array}\right)\right)\right|_{t_{1}=t_{2}=0} \\
& +\left.\frac{\partial^{2}}{\partial t_{1} \partial t_{2}} T \Phi_{d}\left(t_{1}\left(\begin{array}{c}
i J e_{\mu, k, l} \\
0
\end{array}\right)-t_{2}\left(\begin{array}{c}
0 \\
i J e_{\nu, n, m}
\end{array}\right)\right)\right|_{t_{1}=t_{2}=0} \\
& -\left.i \frac{\partial^{2}}{\partial t_{1} \partial t_{2}} T \Phi_{d}\left(t_{1}\left(\begin{array}{c}
e_{\mu, k, l} \\
0
\end{array}\right)-t_{2}\left(\begin{array}{c}
0 \\
i J e_{\nu, n, m}
\end{array}\right)\right)\right|_{t_{1}=t_{2}=0} \\
& -\left.i \frac{\partial^{2}}{\partial t_{1} \partial t_{2}} T \Phi_{d}\left(t_{1}\left(\begin{array}{c}
i J e_{\mu, k, l} \\
0
\end{array}\right)+t_{2}\left(\begin{array}{c}
0 \\
e_{\nu, n, m}
\end{array}\right)\right)\right|_{t_{1}=t_{2}=0} \\
& =-\left.\frac{\partial^{2}}{\partial t_{1} \partial t_{2}} \exp \left(-\frac{i}{2} t_{1} t_{2} \delta_{\mu \nu} \delta^{k n} \delta^{l m}\right)\right|_{t_{1}=t_{2}=0} \\
& +\left.\frac{\partial^{2}}{\partial t_{1} \partial t_{2}} \exp \left(+\frac{i}{2} t_{1} t_{2} \delta_{\mu \nu} \delta^{k n} \delta^{l m}\right)\right|_{t_{1}=t_{2}=0}-0-0 \\
& =i \delta_{\mu, \nu} \delta^{k n} \delta^{l m}
\end{aligned}
$$

For $k \geq l$ and $m \leq N$ we obtain the same. Having the formula (23) in hand on easily proves that in all other cases the propagators are equal to zero.

The computation of a contribution to the partition function $Z$ from the $k$ th order can now be done by summing up Kronecker delta functions, see (15)(18). A systematic formalism to do this is the Feynman diagram technique.

\subsection{Feynman diagrams}

Let us interpret (15)-(18) in terms of the Feynman diagram technique. Each factor in the product (17) is a vertex function. These vertex functions are identified with vertices as illustrated in Figure 3. The propagators $<A_{\mu}^{k l} B_{\nu}^{m n}>$ and $<A_{\mu}^{k l} A_{\nu}^{m n}>$ can be represented by triple lines, see Figure 1 . Each line corresponds to the separate propagation of its two indices. The middle line carries a Greek index $\mu, \nu, \ldots$ and the external lines carry Latin indices. The matrix $A_{\mu}$ corresponds to + and $B_{\mu}$ corresponds to -. 


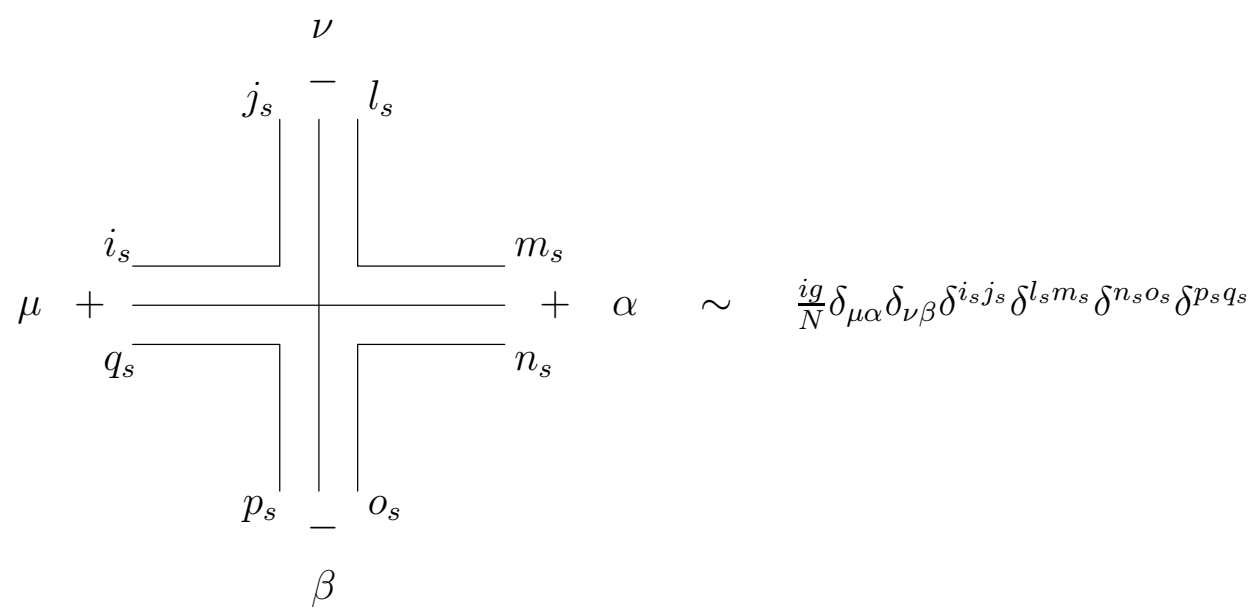

Figure 3: Feynman diagram corresponding to vertex functions

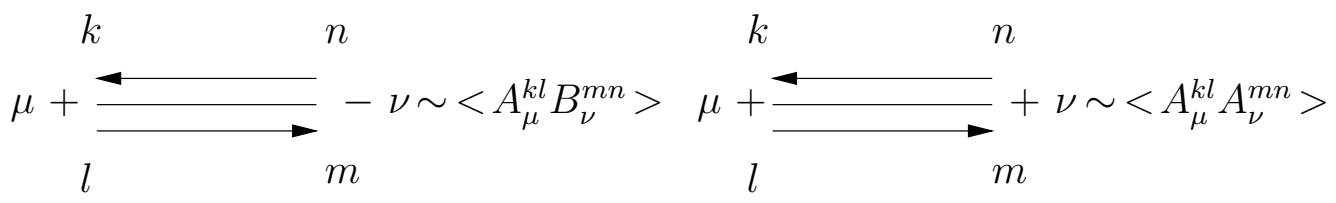

Figure 4: Feynman diagrams corresponding to propagators 
To compute a contribution to the partition function $Z$ from the $k$-th order of perturbation theory we draw diagrams with the $k$ vertices from the $k$-th order of perturbation theory, see (17). Then we have to connect the endpoints of the vertices corresponding to the propagators obtained from the Wick theorem. Doing this we obtain $(4 k) ! /\left(2^{2 k}(2 k) !\right)$ diagrams, if in such a diagram all propagators are different from zero then we call the diagram connected. In Figure 5 this is illustrated for $k=3$ and, additionally, it is explained how to interpret a planar connected Feynman diagram as knot diagram.
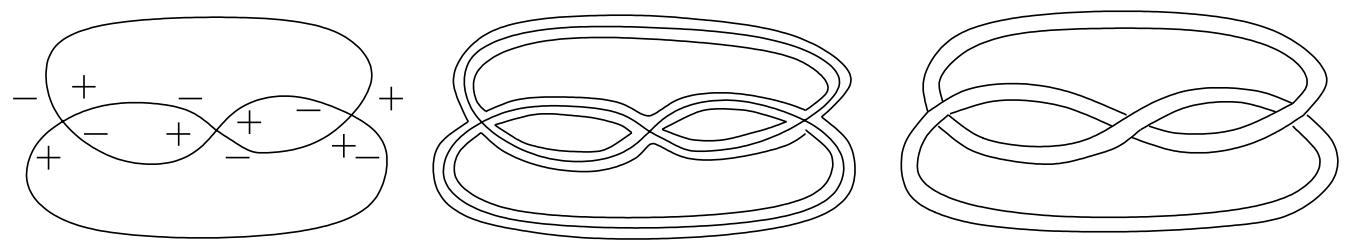

Figure 5: Third order graph (trefoil) in triple line representation

The known connection between planarity and the large $N$ limit t'H74 is based on the Euler theorem. A general Feynman diagram consists of $P$ propagators, $V$ vertices, and $C$ closed loops of Latin indices. The contribution of a connected diagram to the partition function is proportional to $(g / N)^{V} N^{C}=g^{V} N^{C-V}$. This is clear upon analyzing the explicit formula for the perturbation series of the partition function $Z$, see (14) and (15)-(18) ( $C$ closed loops of Latin indices in a connected Feynman diagram cause, in summing up the Kronecker functions in (15)-(18), a contribution $N^{C}$ ). Note that a contribution to the $k$-th order of perturbation theory may consist of one up to $k$ connected diagrams. For a connected diagram one has $P=2 \mathrm{~V}$. Each closed loop of Latin index may be considered as a face of a polyhedron and the Euler relation reads $V-P+C=2-2 p$ where $p=0,1, \ldots$ is the number of holes of the surface on which the polyhedron is drawn (genus of the Riemannian surface). Therefore $C-V=2-2 p$ and the contribution of the diagram is proportional to $g^{V} N^{2-2 p}$. We obtain that the principal contribution comes from the planar diagrams with $p=0$. Now in a similar vine one gets that if a planar diagram has $l$ closed loops with Greek indices (i.e., one has a l-link) then the contribution is of the diagram is proportional to $d^{l}$. In particular, the contribution of the knot diagrams $l=1$ is proportional 
to $d$. Hence, we obtain the following formal expansion

$$
\ln Z(N, d, g)=N \ln 2+d N^{2} \ln \pi+N^{2} \sum_{l=1}^{\infty} \sum_{p=0}^{\infty} F_{l, p}(g) N^{-2 p} d^{l} .
$$

Note that $Z(N, d, 0)$ is real and therefore $\ln Z(N, d, g)$ is uniquely defined as the formal series over $g$. The summand $N \ln 2+d N^{2} \ln \pi$ has its origin in the relation $\Phi_{N, d}=2^{d N} \pi^{d N^{2}} \Phi_{d}$, see Remark 3.8(ii) and Remark 4.4, which reflects the fact that the partition function $Z$ is not normalized. This normalizing constant has not taken into account in [AV98. Having derived (24) except for this summand in AV98 the authors have concluded the statement of Theorem 1.1. As generating function for the alternating knot diagrams they have found $F(g)=F_{1,0}(g)$. Using (24) we obtain $F(g)=\ln \pi+F_{1,0}(g)$. Consider $F_{1,0}$ in its formal series representation in the coupling constant $g$. Then the source of the coefficient of the $k$-th power in $g$ are the planar Feynman diagrams with $k$ vertices and one closed loop of Greek indices. Hence, $F_{1,0}$ can be interpreted as the generating function for the alternating knot diagrams.

Remark 4.6 (i) One has a similar proposition for all (not only for alternating) knot diagrams if one takes the following action:

$$
\begin{aligned}
S(A, B, g) & =\operatorname{Tr}\left(A_{\mu} A_{\mu}\right)+\operatorname{Tr}\left(B_{\mu} B_{\mu}\right) \\
& +\operatorname{Tr}\left(A_{\mu} B_{\mu}\right)+\frac{g}{2 N} \operatorname{Tr}\left(A_{\mu} B_{\nu} A_{\mu} B_{\nu}\right),
\end{aligned}
$$

$A, B \in \operatorname{Mat}_{N}^{h}(\mathbb{C})$.

(ii) The first Reidemeister move in Figure 6 admits a natural interpretation in the Feynman diagram technique for the action (6). This move generates the so called tadpole diagrams, see Figure Q6, and can be removed by using the Wick ordered product if we use the action in the form

$S(A, B, g)=\operatorname{Tr}\left(A_{\mu} B_{\mu}\right)+\frac{g}{2 N}: \operatorname{Tr}\left(A_{\mu} B_{\nu} A_{\mu} B_{\nu}\right):_{\Phi_{N, d}}, A, B \in \operatorname{Mat}_{N}^{h}(\mathbb{C})$,

where

$$
\begin{aligned}
: \operatorname{Tr}\left(A_{\mu} B_{\nu} A_{\mu} B_{\nu}\right):_{\Phi_{N, d}} & :=\sum_{\mu, \nu=1}^{d} \sum_{j, l, m, n=1}^{N}: A_{\mu}^{j l} B_{\nu}^{l, m} A_{\mu}^{m n} B_{\nu}^{n, j}:_{\Phi_{N, d}} \\
& =\operatorname{Tr}\left(A_{\mu} B_{\nu} A_{\mu} B_{\nu}\right)+4 d n \operatorname{Tr}\left(A_{\mu} B_{\mu}\right)+2 d N^{3} .
\end{aligned}
$$



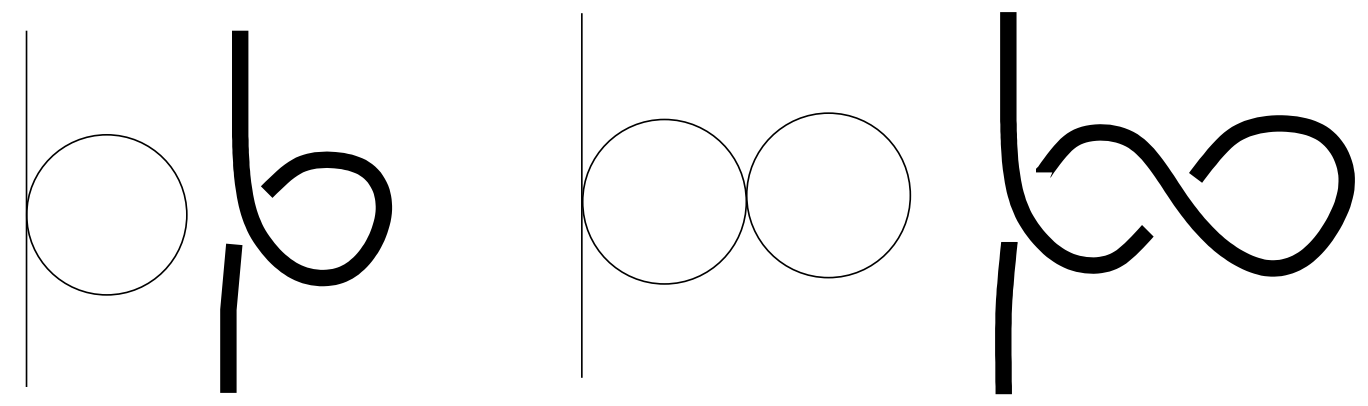

Figure 6: Tadpole diagrams

Here: : $\Phi_{N, d}: \mathcal{P}\left(\right.$ Mat $\left._{N, 2 d}^{h}\right) \rightarrow \mathcal{P}\left(\right.$ Mat $\left._{N, 2 d}^{h}\right)$, maps smooth polynomials to Wick ordered polynomials where the Wick ordering is performed w.r.t. the generalized function $\Phi_{N, d}$.

\section{$5 \quad$ Knots and M(atrix)-theory}

In AV98 the authors have drawn consequences of Theorem 1.1 concerning M-theory. Let us quote their conclusions.

Matrix models of M-theory and superstrings are obtained by the dimensional reduction of super Yang-Mills theory in ten dimensional space-time to $p$ dimensions, $p=0,1,2$, see [BFSS97], [Per97], [IKKT97], and [DVV97. The bosonic part of the action in the matrix approach to M-theory has the form

$$
S=\int_{\mathbb{R}} \frac{1}{2} \operatorname{Tr}\left(\dot{A}_{\mu}(t) \dot{A}_{\mu}\right)(t)+\frac{g}{4 N} \operatorname{Tr}\left(\left[A_{\mu} A_{\nu}\right]\left[A_{\mu} A_{\nu}\right]\right) d t .
$$

Here $A_{\mu}(t)$ are Hermitian $(N \times N)$-matrices over the field $\mathbb{C}$ depending on time and $1 \leq \mu \leq d$. One has

$$
\frac{1}{2} \operatorname{Tr}\left(\left[A_{\mu} A_{\nu}\right]\left[A_{\mu} A_{\nu}\right]\right)=\operatorname{Tr}\left(A_{\mu} A_{\nu} A_{\mu} A_{\nu}\right)-\operatorname{Tr}\left(A_{\mu} A_{\nu} A_{\mu} A_{\nu}\right) .
$$

The first term in (26) has the form which has been discussed in the previous sections. In the limit $d \rightarrow 0$ the principal contribution comes from knot-like diagrams which have one loop with Greek indices. The same reasoning one 
can apply to the IKKT matrix model [IKKT97] with the action

$$
S=\frac{N}{2 g} \operatorname{Tr}\left(\left[A_{\mu} A_{\nu}\right]^{2}\right)
$$

If one makes the assumption on the existence of nonzero condensate $<A_{\mu}, A_{\mu}>\approx 1$ then one gets

$$
S_{e f f}=\frac{N}{2 g}\left(\operatorname{Tr}\left(A_{\mu} A_{\mu}\right)+\operatorname{Tr}\left(A_{\mu} A_{\nu} A_{\mu} A_{\nu}\right)-\operatorname{Tr}\left(A_{\mu} A_{\nu} A_{\mu} A_{\nu}\right)\right)
$$

and one can use the described diagram technique.

Furthermore, in AV98 the authors speculate that Theorem 1.1 and its consequences indicate that perhaps a prototypical M-theory without matrix theory $(d=0)$ exists in the void. The eleven dimensional M-theory could be obtained from this prototypical M-theory by the decompactification of a point.

\section{Acknowledgments}

Financial support of the DFG through the project STR 88/6-1 is gratefully acknowledged. Part of this work was done at CCM under the auspices of PRAXIS XXI (2/2.1/MAT/175/94) with support from FEDER through CITMA. I. Volovich is grateful to L. Streit for the kind hospitality at the Universidade da Madeira, Funchal, where this work was started. 


\section{References}

[AAV96] L. Accardi, I.Ya. Aref'eva, and I.V. Volovich. The master field for half-planar diagrams and free non-commutativ random variables. In V. Matveev and V. Rubakov, editors, Quarks 96, 1996.

[AH76] S. Albeverio and R. Høegh-Krohn. Mathematical Theory of Feynman Path Integrals, volume 523 of Lecture Notes in Mathematics. Springer Verlag, Berlin, Heidelberg, New York, 1976.

[Are83] I.Ya. Aref'eva. Reduced large N models as amorphous systems. Phys. Lett. B, 124:154-159, 1983.

[AS94] S. Albeverio and J. Schäfer. A mathematical model of Ablian Chern-Simons theory. In S. Albeverio, U. Cattaneo, and D. Merlini, editors, Stochastic Processes, Physics and Geometry II, Singapore, 1994. World Scientific.

[AS97] S. Albeverio and A. Sengupta. A mathematical construction of the non-Ablian Chern-Simons functional. Comm. Math. Phys., 186:563-579, 1997.

[Ati90] M. Atiyah. The Geometry and Physics of Knots. Cambridge University Press, Cambridge, 1990.

[AV96] I.Ya. Aref'eva and I.V. Volovich. The master field for QCD and q-deformed quantum field theory. Nucl. Phys. B, 462:600-612, 1996.

[AV98] I.Ya. Aref'eva and I.V. Volovich. Knots and matrix models. Infinite Dim. Anal. Quantum Prob., 1(1):167-173, 1998.

[BFSS97] T. Banks, W. Fischler, S.H. Shenker, and L. Susskind. M theory as a matrix model: a conjecture. Phys. Rev. D, 55:5112-5128, 1997.

[BIPZ78] E. Brézin, C. Itzykson, G. Parisi, and J.-B. Zuber. Planar diagrams. Comm. Math. Phys., 59:35-51, 1978.

[Bir93] J.S. Birman. New point of view in knot theory. Bull. Amer. Math. Soc., 28(2):253-287, 1993. 
[BK95] Yu.M. Berezansky and Yu.G. Kondratiev. Spectral Methods in Infinite-Dimensional Analysis. Kluwer Academic Publishers, Dordrecht, 1995. Originally in Russian, Naukova Dumka, Kiev, 1988.

[BNGRT97] D. Bar-Natan, S. Garoufalidis, L. Rozansky, and D.P. Thurston. Wheels, wheeling, and the Kontsevich integral of the unknot. Preprint q-alg/9703025, xxx.lanl.gov, 1997.

[dG82] P.-G. de Gennes. Scaling Concepts in Polymer Physics. Cornell Univ. Press, Cornell, 1982.

[DVV97] R. Dijkgraaf, E. Verlinde, and H. Verlinde. Matrix string theory. Nucl. Phys. B, 500:43-61, 1997.

[FN97] L. Faddeev and A.J. Niemi. Stable knot-like structures in classical field theory. Nature, 387:58-61, 1997.

[Hid80] T. Hida. Brownian Motion. Springer Verlag, 1980.

[HKPS93] T. Hida, H.-H. Kuo, J. Potthoff, and L. Streit. White Noise. An infinite dimensional calculus. Kluwer, Dordrecht, 1993.

[IKKT97] N. Ishibashi, H. Kawai, Y. Kitazawa, and A. Tsuchiya. A largeN reduced model as superstring. Nucl. Phys. B, 498:467-491, 1997.

[Kau90] L.H. Kauffman. Knots and Physics. World Scientific, Singapore, 1990.

$\left[K \mathrm{CP}^{+} 96\right]$ Yu.G. Kondratiev, P. Leukert, J. Potthoff, L. Streit, and W. Westerkamp. Generalized functionals in Gaussian spaces: The characterization theorem revisited. J. Funct. Anal., 141(2):301-318, 1996.

[Kon80] Yu.G. Kondratiev. Nuclear spaces of entire functions in problems of infinite dimensional analysis. Soviet Math. Dokl., 22:588-592, 1980.

[Kon91] Yu.G. Kondratiev. Spaces of entire functions of an infinite number of variables, connected with the rigging of a Fock space. 
Selecta Mathematica Sovietica, 10(2):165-180, 1991. Originally published in Spectral Analysis of Differential Operators, Mathematical Institute of the Academy of Sciences of the Ukrainian SSR, Kiev, 1980, pp. 18-37.

[KS78] Yu.G. Kondratiev and Yu.S. Samoilenko. Spaces of trial and generalized functions of an infinite number of variables. Rep. Math. Phys., 14(3):325-350, 1978.

[KT80] I. Kubo and S. Takenaka. Calculus on Gaussian white noise I, II. Proc. Japan Acad., 56:376-380 and 411-416, 1980.

[Kuo96] H.-H. Kuo. White Noise Distribution Theory. CRC Press, Boca Raton, New York, London, Tokyo, 1996.

[LS96] P. Leukert and J. Schäfer. A rigorous construction of Ablian Chern-Simons path integrals using white noise analysis. Rev. Math. Phys., 8:445-456, 1996.

[Oba94] N. Obata. White Noise Calculus and Fock Space, volume 1577 of Lecture Notes in Mathematics. Springer Verlag, Berlin, Heidelberg, New York, 1994.

[Per97] V. Periwal. Matrices on a point as the theory of everything. Phys. Rev. D, 55:1711-1713, 1997.

[Pie72] A. Pietsch. Nuclear Locally Convex Spaces. Springer Verlag, Berlin, Heidelberg, New York, 1972.

[PS91] J. Potthoff and L. Streit. A characterization of Hida distributions. J. Funct. Anal., 101:212-229, 1991.

[Sch71] H.H. Schaefer. Topological Vector Spaces. Springer Verlag, Berlin, Heidelberg, New York, 1971.

[Sch91] J. Schäfer. Abelsche Chern-Simons Theorie. Master's thesis, Universität Bochum, 1991.

[t'H74] G. t'Hooft. A planar diagram theory for strong interactions. Nucl. Phys. B, 72:461-473, 1974. 
[Wit89] E. Witten. Quantum field theory and the Jones polynomial. Comm. Math. Phys., 121:351-399, 1989. 